\title{
Recurrent homozygous deletion of DROSHA and microduplication of PDE4DIP in pineoblastoma
}

Matija Snuderl1,2,3,4, Kasthuri Kannan (10 1,4, Elke Pfaff5,6,7, Shiyang Wang ${ }^{8}$, James M. Stafford ${ }^{9}$, Jonathan Serrano ${ }^{10}$, Adriana Heguy $2,4,10$, Karina Ray ${ }^{10}$, Arline Faustin ${ }^{3,4}$, Olga Aminova ${ }^{10}$, Igor Dolgalev (1) 4,10,

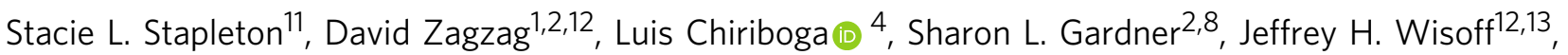
John G. Golfinos ${ }^{12}$, David Capper ${ }^{14,15,22}$, Volker Hovestadt ${ }^{16}$, Marc K. Rosenblum ${ }^{17}$, Dimitris G. Placantonakis ${ }^{11,18}$, Sarah E. LeBoeuf ${ }^{4}$, Thales Y. Papagiannakopoulos ${ }^{4}$, Lukas Chavez (1) ${ }^{6}$, Sama Ahsan ${ }^{19}$, Charles G. Eberhart ${ }^{20}$, Stefan M. Pfister ${ }^{5,6,7}$, David T.W. Jones ${ }^{5,6}$ \& Matthias A. Karajannis (1) 21

Pineoblastoma is a rare and highly aggressive brain cancer of childhood, histologically belonging to the spectrum of primitive neuroectodermal tumors. Patients with germline mutations in DICER1, a ribonuclease involved in microRNA processing, have increased risk of pineoblastoma, but genetic drivers of sporadic pineoblastoma remain unknown. Here, we analyzed pediatric and adult pineoblastoma samples $(n=23)$ using a combination of genomewide DNA methylation profiling and whole-exome sequencing or whole-genome sequencing. Pediatric and adult pineoblastomas showed distinct methylation profiles, the latter clustering with lower-grade pineal tumors and normal pineal gland. Recurrent variants were found in genes involved in PKA- and NF-KB signaling, as well as in chromatin remodeling genes. We identified recurrent homozygous deletions of DROSHA, acting upstream of DICER1 in microRNA processing, and a novel microduplication involving chromosomal region 1q21 containing PDE4DIP (myomegalin), comprising the ancient DUF1220 protein domain. Expresion of PDE4DIP and DUF1220 proteins was present exclusively in pineoblastoma with PDE4DIP gain.

\footnotetext{
${ }^{1}$ Division of Neuropathology, NYU Langone Health, New York 10016 NY, USA. ${ }^{2}$ Laura and Isaac Perlmutter Cancer Center, NYU Langone Health, New York 10016 NY, USA. ${ }^{3}$ Department of Neurology, NYU Langone Health, New York 10016 NY, USA. 4 Department of Pathology, NYU Langone Health, New York 10016 NY, USA. ${ }^{5}$ Hopp Children's Cancer Center at the NCT Heidelberg (KiTZ), Heidelberg 69120, Germany. ${ }^{6}$ Division of Pediatric Neurooncology, German Cancer Research Center (DKFZ), Heidelberg 69120, Germany. ${ }^{7}$ Department of Pediatric Oncology, Hematology \& Immunology, Heidelberg, University Hospital, Heidelberg 69120, Germany. ${ }^{8}$ Division of Pediatric Hematology/Oncology, NYU Langone Health, New York 10016 NY, USA. ${ }^{9}$ Department of Biochemistry and Molecular Pharmacology, NYU Langone Health, New York 10016 NY, USA. ${ }^{10}$ Genome Technology Center, NYU Langone Health, New York 10016 NY, USA. ${ }^{11}$ Johns Hopkins All Children's Hospital, Cancer and Blood Disorders Institute, St Petersburg 33701 FL, USA. ${ }^{12}$ Department of Neurosurgery, NYU Langone Health, New York 10016 NY, USA. ${ }^{13}$ Division of Pediatric Neurosurgery, NYU Langone Health, New York 10016 NY, USA. ${ }^{14}$ Department of Neuropathology, Institute of Pathology, University of Heidelberg, Heidelberg 69120, Germany. ${ }^{15}$ Clinical Cooperation Unit Neuropathology, German Cancer Consortium (DKTK), German Cancer Research Center (DKFZ), Heidelberg 69120, Germany. ${ }^{16}$ Division of Molecular Genetics, German Cancer Research Center (DKFZ), Heidelberg 69120, Germany. ${ }^{17}$ Department of Pathology, Memorial Sloan Kettering Cancer Center, New York 10065 NY, USA.

${ }^{18}$ Neuroscience Institute and Kimmel Center for Stem Cell Biology, NYU Langone Health, New York 10016 NY, USA. ${ }^{19}$ Division of Pediatric Oncology, Johns Hopkins Hospital, Baltimore 21218 MD, USA. ${ }^{20}$ Department of Pathology, Johns Hopkins Hospital, Baltimore 21218 MD, USA. ${ }^{21}$ Department of Pediatrics, Memorial Sloan Kettering Cancer Center, New York 10065 NY, USA. ${ }^{22}$ Present address: Charité - Universitätsmedizin Berlin, corporate member of Freie Universität Berlin, Humboldt-Universität zu Berlin, and Berlin Institute of Health, Department of Neuropathology, Berlin 10117, Germany. These authors contributed equally: Matija Snuderl, Kasthuri Kannan. Correspondence and requests for materials should be addressed to M.A.K.(email: karajanm@mskcc.org)
} 
ccording to the 2016 World Health Organization (WHO) Classification of Tumors of the Central Nervous System, tumors of the pineal region comprise pineocytoma (grade I), pineal parenchymal tumor of intermediate differentiation (PPTID) (grade II or III), pineoblastoma (PB) (grade IV), and papillary tumor of the pineal region (grade II or III) ${ }^{1-3}$. PBs are very rare tumors, accounting for less than $1 \%$ of pediatric brain tumors, and our current knowledge of the molecular genetics and biology of PB is limited.

Histologically, PB closely resembles other "small round blue cell" embryonal tumors or primitive neuroectodermal tumors (PNET), such as medulloblastoma and retinoblastoma in the brain, and Ewing sarcoma/peripheral PNET, and neuroblastoma in the bone and adrenal gland, respectively. While the majority of $\mathrm{PB}$ appear to arise sporadically, known predispositions include germline mutations in the RB1 and DICER1 genes ${ }^{1,2}$. A subset of $\mathrm{PBs}$ arise in the setting of familial bilateral retinoblastoma, which is then termed trilateral retinoblastoma syndrome ${ }^{3}$. PBs have also been reported in patients with familial adenomatous polyposis ${ }^{3}$.

Genetic drivers of sporadic PBs are unknown. Despite morphological similarities, $\mathrm{PBs}$ do not seem to share any of the recurrent genetic drivers identified in medulloblastoma or in "CNS-PNET"4. Previous studies in PB have identified recurrent losses of chromosome $22 \mathrm{q}$, as well as high level gains of $1 \mathrm{q} 12$, $5 \mathrm{p} 13,5 \mathrm{q} 21,6 \mathrm{p} 12$, and $14 \mathrm{q} 21^{1}$, and microarray studies showed significant transcriptional upregulation of UBEC2, SOX4, TERT, TEP1, PRAME, CD24, POU4F2, and HOXD13 $3^{5,6}$. In this study we sought to identify molecular drivers of sporadic $\mathrm{PB}$ to gain insights into the biology of this rare disease.

Here, we show that adult and pediatric PBs have different methylation profiles and harbor recurrent homozygous deletions of DROSHA, as well as microduplication of PDE4DIP containing the ancient DUF1220 domain, representing novel molecular drivers of $\mathrm{PB}$. Furthermore, heterogeneity within the pediatric $\mathrm{PB}$ methylation cluster indicates the presence of biological subgroups within pediatric $\mathrm{PB}$. Larger retrospective and prospective studies will be necessary to further delineate the diagnostic, prognostic, and therapeutic relevance of our findings for children afflicted with this rare cancer.

\section{Results and Discussion}

Genome-wide DNA methylation profiling. To ascertain whether PBs and PPTID can be distinguished using genome-wide DNA methylation profiling, as shown for other pediatric brain tumors $^{4,7}$; we performed unsupervised hierarchical clustering selecting the 5000 probes that showed the highest standard deviation (SD) across our samples. We compared 10 samples from normal autopsy pineal glands (average age 61 years), 16 tumors clinically and histologically diagnosed as $\mathrm{PB}$ and five tumors diagnosed as PPTID. Of 16 PBs, 13 formed a distinct cluster, while two tumors clustered with PPTID (PB3 and PB8) and one tumor (PB23) clustered closer to normal pineal tissue (Fig. 1). All three tumors were histologically and clinically confirmed as PBs. Strikingly, review of the clinical information showed that all three tumors were PB that arose in adults. Sample PB23, which clustered closely with the normal pineal gland group, albeit with noticeable differences (Fig. 1), was from a 69-year-old patient, the oldest in our group (Supplementary Fig. 1). Patients with PBs clustering with the PPTID samples were 45 (PB3) and 37 (PB8) years old at the time of diagnosis. This may suggest that $\mathrm{PBs}$ presenting in adults may arise from preexisting PPTIDs or normal pineal gland (secondary $\mathrm{PB}$ ), whereas pediatric $\mathrm{PB}$ might arise directly from embryonal neuroblasts (primary $\mathrm{PB}$ ). However, this needs to be confirmed in larger studies, including comparison with high-grade PPTID. An alternative explanation is that at least some of the PBs in adults may actually represent misclassified pineal parenchymal tumors of intermediate differentiation; however, the diagnosis of $\mathrm{PB}$ was rendered by four experienced board certified neuropathologists.

Genome sequencing. To identify candidate oncogenic drivers in $\mathrm{PB}$, we performed whole-exome sequencing (WES) on $13 \mathrm{~PB}$ without available matching germline DNA and whole-genome sequencing (WGS) on five PB samples with matched normal germline DNA (Fig. 2, Table 1). Out of five WGS, two were primary $\mathrm{PBs}$ and three were synchronous metastases (M1-M3) from one of the WGS tumors (PB1) obtained after tumor recurrence during autopsy. Due to the small size of the samples, only eight tumors had sufficient DNA for validation of the identified mutations (Fig. 2). Mean tumor target coverage was $174 \times$ for WES and $33 \times$ for WGS. Mean normal target coverage for WGS was 36×. On average, we identified 1545 nonsynonymous putative mutations (WES) and 74 nonsynonymous somatic mutations in 51 genes (WGS) in exons. Similarly to other pediatric cancers, overall mutational burden in PB was relatively low (Supplementary Fig. 2), similar to other pediatric cancers.

In WGS samples, the primary tumor and metastatic foci shared somatic missense mutations in MGLL, GPX5, and COL2A1, while all three metastatic foci shared mutations in ZNF789, TET1, DCHS2, and ACOT6, which were not present in the primary tumor, suggesting these may be associated with the tumor progression or metastasis (Fig. 2). Interestingly, although PB1 and its metastases shared mutations, all three metastatic lesions showed a much higher mutation rate and had a large number of private mutations (Supplementary Fig. 2a, b). Since the metastatic lesions were collected at the time of autopsy after radiation therapy and myeloablative high-dose chemotherapy, the increased mutational burden may represent at least in part the mutagenic effect of therapy, rather than a natural progression of the disease. Two primary PB, PB1 and PB12, did not share any somatic mutations. (Fig. 2). In WES samples, where only tumor tissue without matched germline DNA was available, we found enrichment by pathway analysis for mutations in the PKA/NFkB signaling pathway $(P T K 2 B, M A P 2 K 7, C R E B B P)$, genes involved in chromatin binding (SREBF2, AGO3, POU1F1, TOP2B) and other pathways (CAPN1, ARRB2, CHD8, SETD8). The most commonly mutated gene in $\mathrm{PBs}$ was $A R R B 2$, with mutations present in eight tumor samples with a hotspot in Arrestin_N domain (Supplementary Fig. 3). MAP2K7, SREBF2, and CAPN1 mutations were present concurrently in three WES samples and appeared to cluster in hotspots (Fig. 2, Supplementary Fig. 3 and Supplementary Table 1). However, due to the unavailability of matching germline DNA from our samples, future studies are necessary to confirm the role of these mutations in $\mathrm{PB}$. In one $\mathrm{PB}$ analyzed by WES (PB15), we could not identify any relevant mutations by pathway enrichment analysis (Fig. 2); however, the tumor showed aberrations in the copy-number profile affecting chromosomes 4, 8, 17, and 22 (Supplementary Fig. 4). Overall, copy-number profiles of pediatric PBs showed widespread chromosomal losses and gains, in contrast to PPTID and PBs of adults, which harbored few chromosomal gains or losses (Supplementary Fig. 4).

Germline and somatic mutations in DICER1 have been recognized as oncogenic drivers in $\mathrm{PB}^{1}$. Germline mutations in DICER 1 are associated with a distinct autosomal dominant tumor predisposition syndrome (OMIM \#601200), whereby patients have an increased risk of developing a variety of extremely rare tumors, such as pleuropulmonary blastoma and PBs. However, DICER1 is associated with susceptibility to $\mathrm{PBs}$ in only a small number of PBs. Interestingly, we did not observe any DICER1 


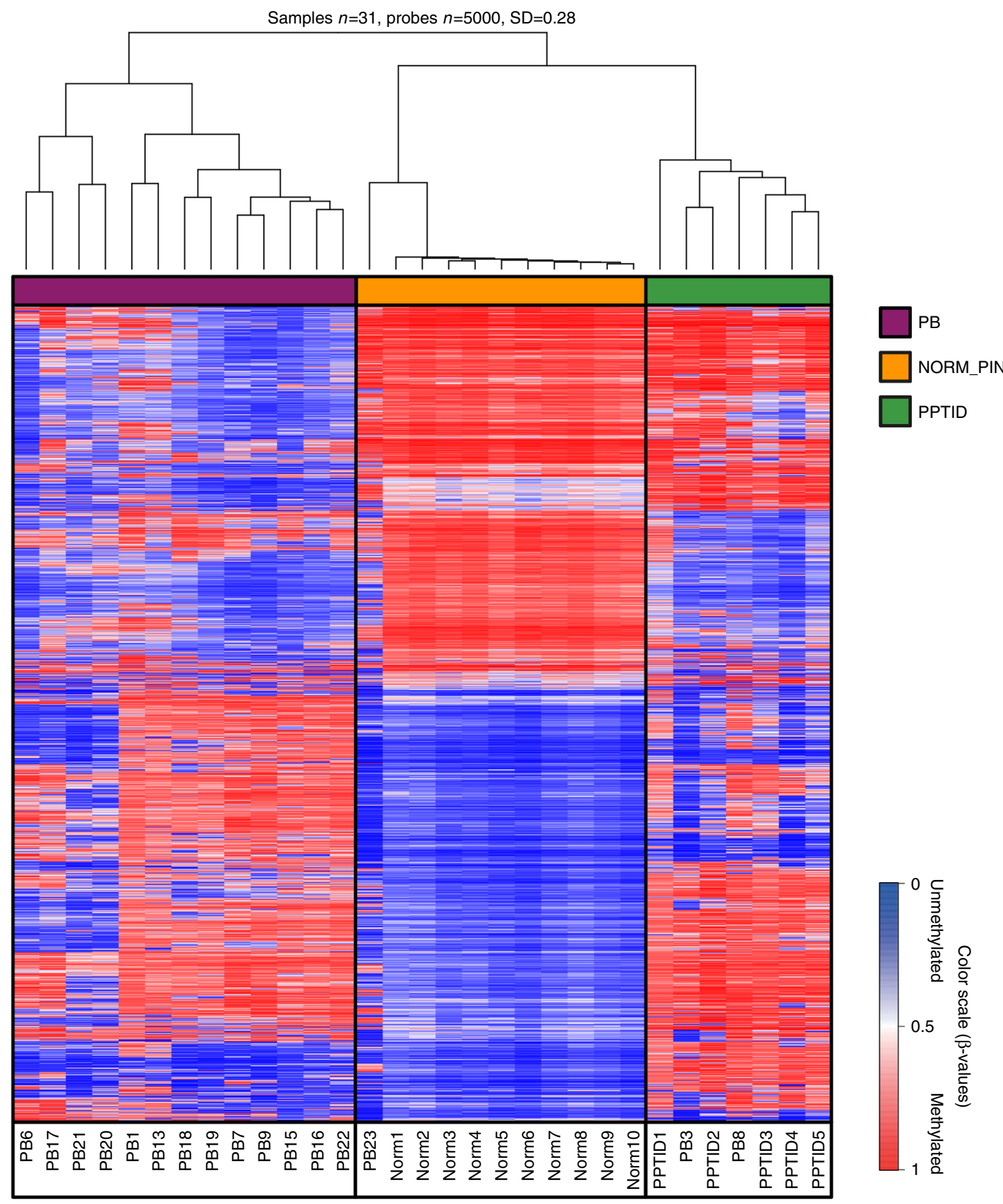

Fig. 1 Methylation signature of pineoblastoma, pineal gland, and PPTID. Pediatric pineoblastomas form a distinct cluster that is separated from normal pineal gland and pineal parenchymal tumor of intermediate differentiation (PPTID). However, adult pineoblastomas cluster with PPTID (PB3 and PB8) as well as the normal pineal gland group (PB23), although analyzed tissues were confirmed as pineoblastoma, suggesting that methylation signature reflects origin of adult pineoblastomas from a normal pineal gland or a lower grade precursor lesion

mutations in our samples despite excellent coverage by both WES and WGS (Supplementary Fig. 5). However, we identified homozygous deletions of DROSHA in 5 out of 19 samples (26\%) (Figs. 2 and 3). DROSHA plays a critical role in miRNA processing in the nucleus, upstream of DICER1, therefore likely resulting in impaired miRNA biogenesis similarly to Wilms tumors, which carry both DROSHA and DICER1 mutations ${ }^{8,9}$. In contrast to Wilms tumor, where DROSHA as well as DICER1 are inactivated by hotspot mutations, we show that in PB DROSHA, similar to DICER $1^{1,10}$, is inactivated by copy-number loss. Within the context of a relatively low mutation burden, this suggests that
PB may be predominantly driven by recurrent copy-number alterations rather than mutations, although further genetic studies with paired tumor-normal are needed.

Further, using WGS, we identified copy-number gains of the gene coding for phosphodiesterase $4 \mathrm{D}$ interacting protein (PDE4DIP) in both primary tumor samples and two out of three metastases (M1 and M3) compared to neighboring 1q21 genes (Fig. 3b) Until recently, the latest human genome build (hg19) annotated a single copy of PDE4DIP per chromosome. Using a haploid human genome, O'Bleness et al. ${ }^{11}$ have since shown that the 1q21 region may contain up to 3 copies of PDE4DIP. Our five 


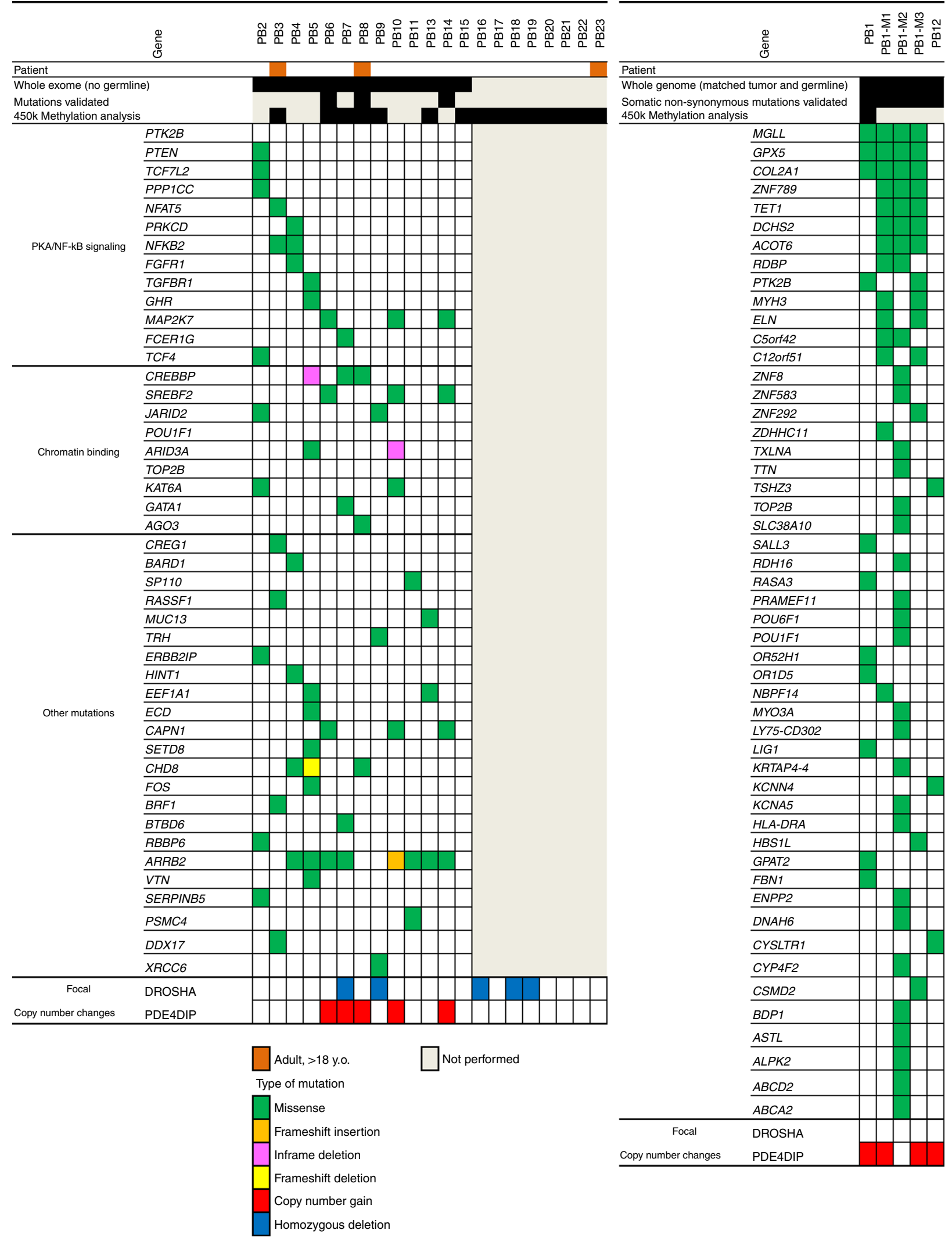

Fig. 2 Genomic landscape of pineoblastoma. Whole-exome sequencing was performed in 13 tumors with no matching germline (exome samples, left). Whole-genome sequencing (genome samples, right) was performed in five tumors, two primary (PB1 and PB12) with matched peripheral blood DNA and three metastasis from PB1 (M1, M2, M3). Specific genes are shown that bear nonsynonymous mutations. In exome samples only mutations enriched in pathway analysis via Ingenuity Pathway Analysis (IPA) software are included. In genome samples, only somatic nonsynonymous mutations are presented. Sufficient material was available to confirm mutations in eight tumors (mutations validated). See also Supplementary Table 1 for mutation details 
WGS tumor datasets showed eight copies of PDE4DIP on average, which would represent a microduplication. When we analyzed our WES samples by confirmatory droplet digital PCR (ddPCR) for PDE4DIP, the average PDE4DIP copy number was significantly higher in additional five $\mathrm{PBs}$ compared to normal pineal tissue controls (paired $t$-test, $p<0.001$ ) (Fig. 3c). These gains were specific to $P D E 4 D I P$, and did not involve neighboring genes in the 1q21 region. In total, we identified gains of PDE4DIP in 7 out of $15 \mathrm{PBs}$ for which sufficient DNA for confirmatory ddPCR was available. In cases where only DNA methylation was performed (PB16-23), PDE4DIP status could not be reliably confirmed due to the insufficient coverage by the $450 \mathrm{k}$ array in this region.

PDE4DIP, myomegalin, is a protein involved in cardiac muscle contraction and suggested to play a role in cardiomyopathy ${ }^{12}$. PDE4DIP is also among the genes affected by 1q21.1 microdele- tion and 1q21.1 microduplication syndromes ${ }^{13}$. DUF1220 domains are interspersed throughout the 1q21 region, including within the PDE4DIP gene ${ }^{11}$. DUF1220 protein domains are approximately 65 amino acids in length and have undergone rapid and extensive copy-number expansion during mammalian evolution, and most strikingly in primate evolution including Homo species ${ }^{14-16}$. DUF1220 domains show the largest humanlineage-specific increase in copy number of any protein-coding region in the human genome. While humans carry $\sim 289$ copies of DUF1220, the copy number is significantly lower in other species, ranging from 125 in chimpanzees to 99 in gorillas, 4 in dolphins, 1 copy in mice, and 0 in non-mammals ${ }^{11,14,15}$. DUF1220 sequences exist within two distinct genomic environments. A single so-called ancestral domain is located in the PDE4DIP gene. The DUF1220 domain within PDE4DIP gene is present in primates, as well as other mammals. Other DUF1220

\section{Table 1 Overview of whole-genome (WGS) and whole-exome (WES) sequencing of pineoblastoma}

\section{Statistic}

Tumor/tumor-normal pairs sequenced

Total Gb sequenced

Mean fold tumor target coverage

Mean fold normal target coverage

Mean number of exonic variants/mutations identified

Mean number of nonsynonymous variants/mutations identified

Mean number of synonymous variants/mutations identified

Total number of validated nonsynonymous variants/mutations

\begin{tabular}{ll} 
Whole exome & Whole genome \\
\hline 13 & $5(2$ primary +3 mets $)$ \\
(tumor only) & (tumor + matched germline) \\
144.1 & 15.0 \\
174.4 & 32.8 \\
- & 36.4 \\
1544.9 & 121.4 \\
522.3 & 83.6 \\
795.2 & 31.8 \\
137 & 74
\end{tabular}
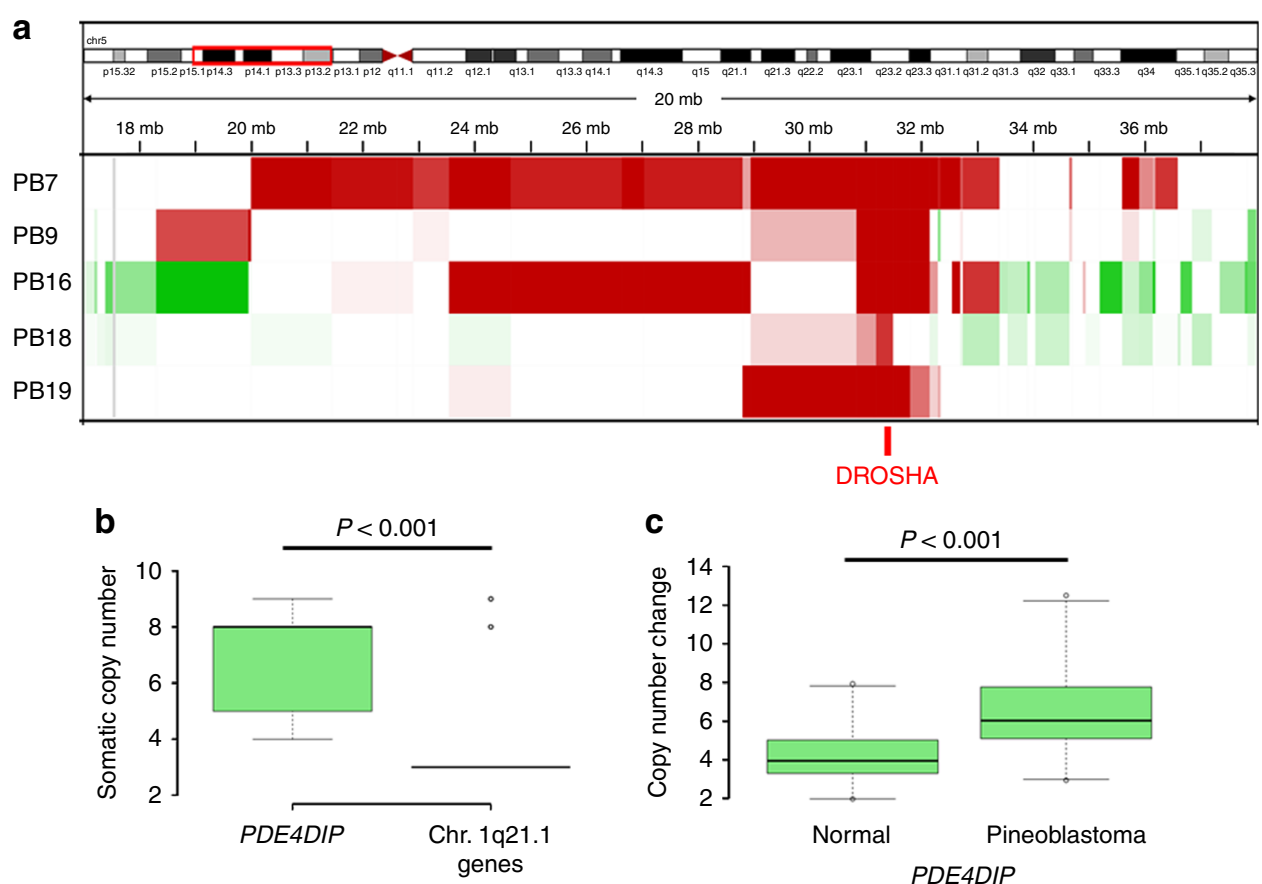

Fig. 3 Focal copy-number aberrations in pineoblastoma. a Homozygous deletions of DROSHA were identified in five pediatric pineoblastomas. For four out of five cases with DROSHA deletion, the deletion is about 1.300-3.755 kb including the whole DROSHA gene. For one case, the deletion seems to be only $300 \mathrm{~kb}$, spanning exons $11-35$ of DROSHA. b, c We identified microduplication of PDE4DIP in seven pineoblastomas. b Whole-genome sequencing ( $n=2$ ) shows higher than expected copy number difference between PDE4DIP and neighboring genes in 1q21.1 region in pineoblastomas ( $t$-test $p$ value $<0.001$ ). c Copy number of PDE4DIP in pineoblastoma is significantly increased compared to the number of PDE4DIP copies in normal DNA by ddPCR (average number of PDE4DIP copies in pineoblastoma six, vs. four in normal controls, paired $t$-test between normal and tumors $<0.001)$. Genes neighboring $P D E 4 D I P$ were not amplified by ddPCR confirming that the microduplication was limited to PDE4DIP only 
domains exist as multiple tandem copies in the NBPF genes, which are typically interspersed throughout the 1q21 region. Copy-number changes in DUF1220 appear to be directly linked to pathologic conditions affecting growth and function of the human brain. The number of DUF1220 copies in vertebrates also directly correlates with the number of cortical neurons ${ }^{14}$. Since DUF1220 plays a critical role in proliferation of neuroblasts during brain development and $\mathrm{PB}$ is a neuroectodermal tumor composed of primitive neuroblasts, we sought to establish whether the copy-number gain of PDE4DIP gene is associated with an increase of PDE4DIP and DUF1220 proteins. All PBs with PDE4DIP copy-number gain $(n=7)$ showed markedly increased expression of both PDE4DIP as well as DUF1220 proteins (Fig. 4), while normal pineal gland and adult brain were negative. Further evaluation of normal brain tissues revealed that neuroblasts in the germinal matrix of the fetal brain strongly express DUF1220, as would be expected from its presumed role in rapid neuroblastic proliferation; however, these neuroblasts did not express PDE4DIP protein. We did not observe overexpression of PDE4DIP or DUF1220 proteins in PBs without PDE4DIP microduplication $(n=5)$, confirming that genomic PDE4DIP gain is directly linked to the DUF1220 overexpression in PB

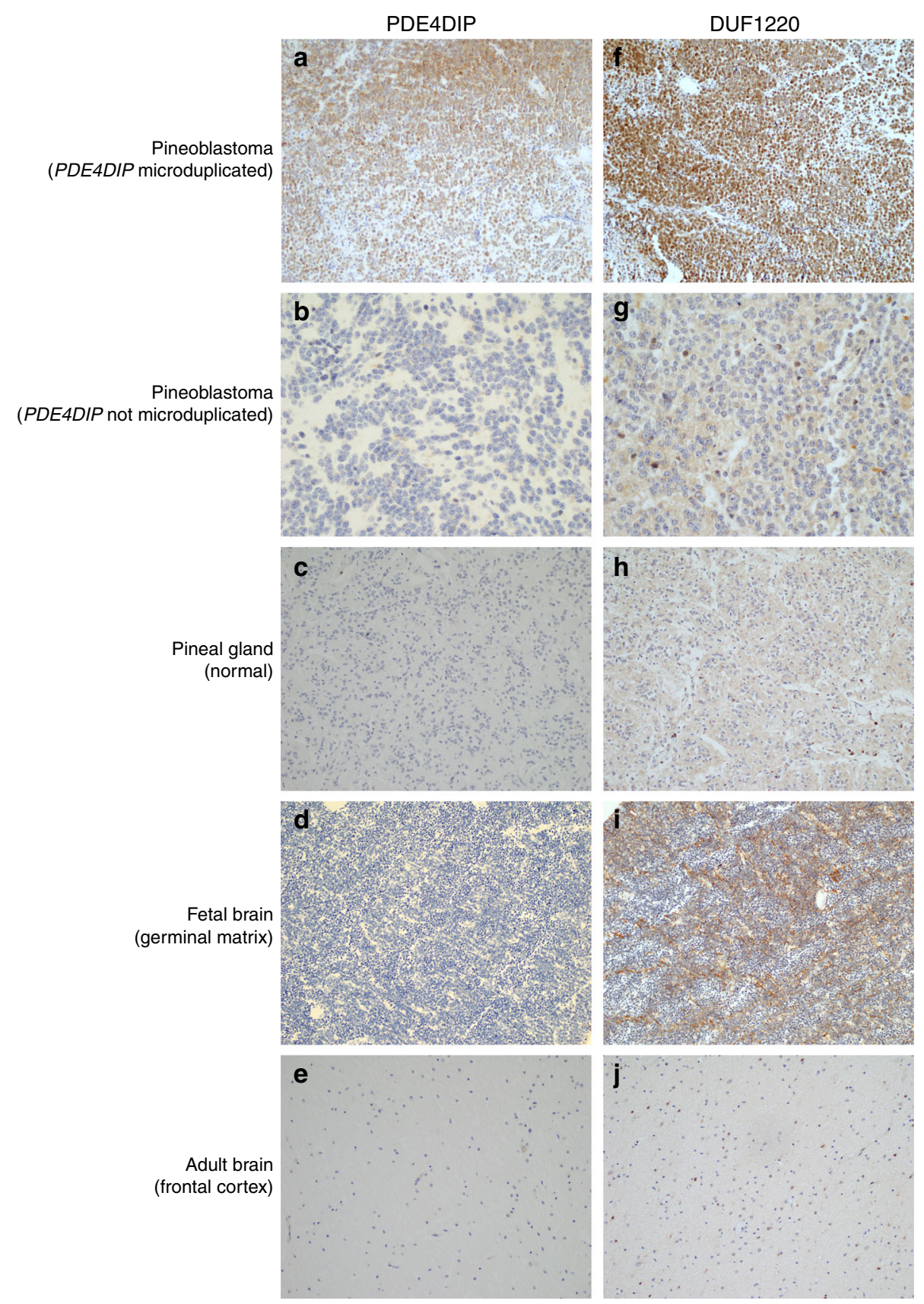

Fig. 4 PDE4DIP microduplication correlates with overexpression of DUF1220. Pineoblastomas with PDE4DIP microduplication ( $n=7)$ show strong expression of both PDE4DIP protein (a), as well as DF1220 protein (f), while pineoblastomas with no PDE4DIP microduplication $(n=5)(\mathbf{b}, \mathbf{g})$ and normal pineal gland ( $\mathbf{c}, \mathbf{h})$ are negative for both proteins. DUF1220 is strongly expressed in the germinal matrix of the fetal brain (i), consistent with its established role in human brain development; however, fetal brain neuroblasts are completely negative for PDE4DIP expression (d). Adult brain tissue is negative for both PDE4DIP and DUF1220 protein expression $(\mathbf{e}, \mathbf{j}) .(n=5$ for each normal control group, original magnification $400 \times$, Scale bar $100 \mu \mathrm{m})$ 
a
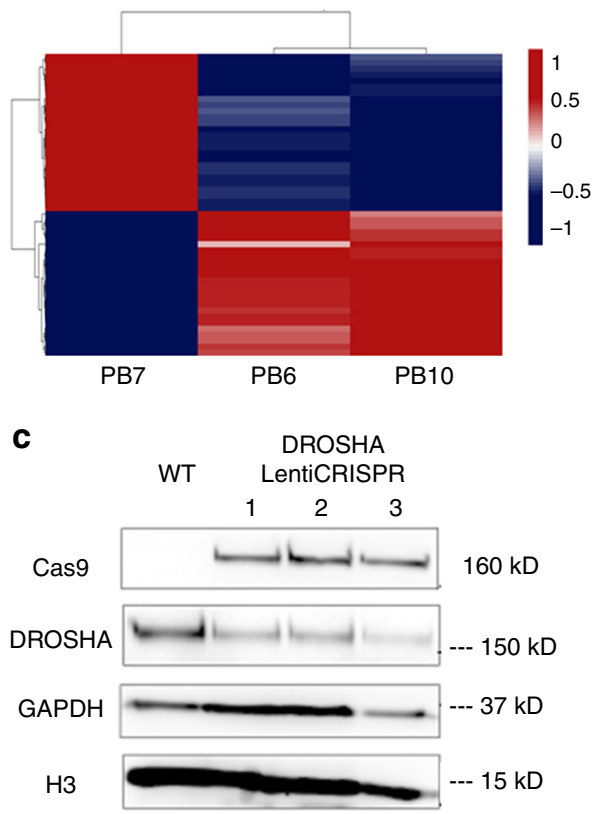

b

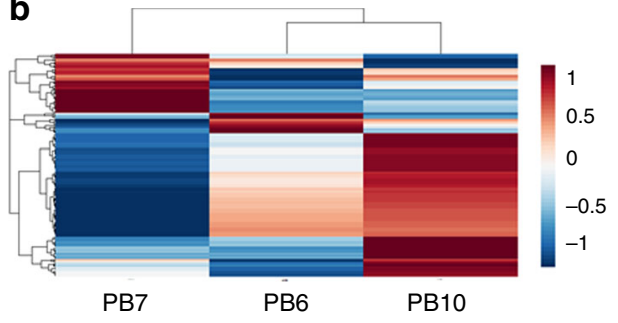

d

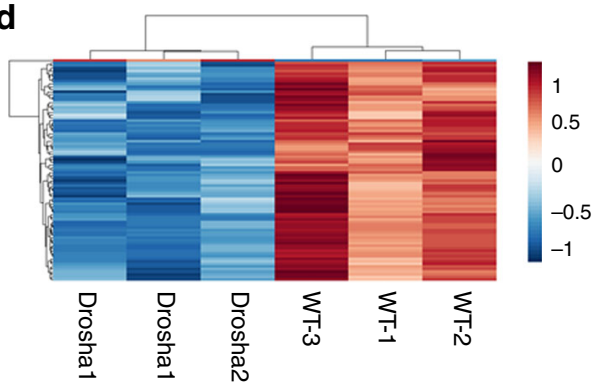

e

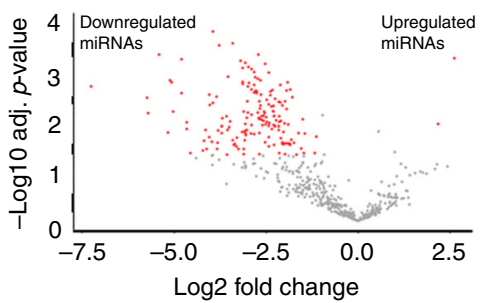

Fig. 5 Effect of DROSHA alteration in pineoblastoma: Three human samples were available for RNA analysis. PB7 with concurrent homozygous DROSHA deletion and PDE4DIP gain, and PB6 and PB10 with PDE4DIP gain only. a Whole-transcriptome analysis shows that PB7 has distinctly different transcriptomic profile compared to PB6 and PB10 (unsupervised clustering, top 50 differentially up and down regulated genes shown), for detailed list see Supplementary Table 2. b PB7 with DROSHA deletion also shows markedly altered miRNA profile, (unsupervised clustering, top 200 differentially expressed miRNA shown, for detailed list see Supplementary Table 3). c Disruption of the DROSHA locus using CRISPR in human neural stem cells (hNSC) shows reduced levels of DROSHA protein, for quantification see Supplementary Fig. 6. d, e hNSC with mutation of DROSHA show markedly reduced levels of miRNA mirroring the clinical samples. d Unsupervised hierarchical clustering analysis based on expression data for the Top 85 differentially expressed miRNA show striking difference between DROSHA-mutated (Drosha1-3) and wild-type (WT1-3) hNSC samples. e Volcano plot shows marked reduction of miRNA in DROSHA-mutated hNSC. The $x$ axis represents the difference of group means of DROSHA mutated and wild-type hNSC; the $y$ axis represents the statistical significance. Each miRNa is represented by a dot, and red dots represent miRNAs that were significantly differentially expressed between groups. For detailed list of miRNAs see also Supplementary Table 4

(Fig. 4). Since the copy-number gain we detected in $1 \mathrm{q} 21$ was limited to PDE4DIP while neighboring regions showed a normal copy number, we concluded that the increase of DUF1220 protein in PBs is due to the copy-number gain of the ancient DUF1220 domain within PDE4DIP, and unlikely due to alterations in the $N B P F$ gene group, where we did not detect any copy-number gains or mutations.

Transcriptome analysis. To evaluate transcriptional differences between the effect of homozygous DROSHA deletion and PDE4DIP microduplication, we performed whole-transcriptome analysis of three PB cases where sufficient quantity and quality of RNA could be isolated from the formalin-fixed paraffin-embedded tissue (FFPE) samples. Two cases (PB6 and PB10) had gain of $P D E 4 D I P$, while one case (PB7) had concurrent gain of PDE4DIP and homozygous loss of DROSHA. Whole-transcriptome analysis showed that homozygous loss of DROSHA leads to distinct changes in RNA expression profile, compared to PDE4DIP gain only (Fig. 5a and Supplementary Table 2). We also performed miRNA profile analysis on clinical samples, confirming that the miRNA profile is markedly affected by loss of DROSHA (Fig. 5b and Supplementary Table 3). However, these analyses need to be confirmed in a larger cohort of PB samples from patients with different ages and underlying mutations. To evaluate the effect of DROSHA mutation experimentally, we disrupted the DROSHA locus in human neural stem cells (hNSC) using the CRISPR/ Cas9 system, leading to decrease of the DROSHA protein, and massive loss of miRNAs in DROSHA-mutated hNSC cell lines (Fig. 5c-e, Supplementary Fig. 6 and Supplementary Table 4), consistent with previous observations of DROSHA and DICER1 effect on miRNA ${ }^{10,17}$.

Conclusions. In summary, we show that pediatric and adult PBs have distinct methylation profiles, suggesting that pediatric tumors arise de novo, whereas adult PBs may arise from a pineal parenchymal tumor or a normal pineal gland. We identified recurrent variants in several oncogenic signaling pathways including PKA, NFKB, and chromatin binding genes, as well as homozygous deletions of DROSHA, but no mutations in DICER1, suggesting that at least two different paths disrupting miRNA processing are involved in the pathogenesis of these tumors. Lastly, we identified a novel microduplication of PDE4DIP 
leading to upregulation of DUF1220 protein. The critical role of DUF1220 in proliferation of neuroblasts during normal embryonal brain development suggests DUF1220 as a novel oncogenic driver in $\mathrm{PB}$.

\section{Methods}

Tissue collection. Tumor, normal pineal gland, and control brain tissues were obtained from the archives of the Departments of Neuropathology, New York University (NYU) Langone Medical Center and School of Medicine, and Johns Hopkins University. This study was approved by the Institutional Review Board of NYU Langone Medical Center (IRB \#14-00948), and informed consent was obtained from study patients in accordance with all insititutional requirements. All specimens were reviewed and classified according to the World Health Organization (WHO) classification ${ }^{3}$ to confirm histological diagnosis and select blocks for DNA extraction and immunohistochemistry. Available clinical data are summarized in the Supplementary Table 5.

DNA extraction. DNA was extracted from FFPE tissue. Areas with the highest available tumor content were chosen. Extraction was carried out using the automated Maxwell system (Promega, Madison, USA).

Whole-exome and whole-genome sequencing and analysis. Exome sequencing: $250 \mathrm{ng}$ of DNA from each sample were sheared on a Covaris instrument for $360 \mathrm{~s}$ (Duty cycle $-10 \%$; intensity -5 ; cycles/Burst -200 ). Barcoded libraries were prepared using the Kapa Low-Throughput Library Preparation Kit Standard (Kapa Biosystems), amplified using the KAPA HiFi Library Amplification kit (Kapa Biosystems) (eight cycles) and quantified using Qubit Fluorimetric Quantitation (Invitrogen) and Agilent Bioanalyzer. An equimolar pool of the four barcoded libraries (300 ng each) was used as input to capture the exome using one reaction tube of the Nimblegen SeqCap EZ Human Exome Library v3.0 (Roche, cat \# 06465684001), according to the manufacturer's protocol. The pooled capture library was quantified by Qubit (Invitrogen) and Bioanalyzer (Agilent), and sequenced on an Illumina HiSeq 2500 using a paired end, 100 nucleotides in length run mode to achieve an average of $100 \times$ coverage.

Whole-genome sequencing: $500 \mathrm{ng}$ of DNA from each sample were sheared on a Covaris instrument as described above, and barcoded libraries were prepared using the Kapa Low-Throughput Library Preparation Kit Standard (Kapa Biosystems), without any PCR amplification.

Fastq reads from both exome and genome sequencing were aligned to human genome build GRCh 37 using BWA ${ }^{18}$ and recalibrated using GATK ${ }^{19}$. In the case of WGS, mutations were detected via Somatic Sniper ${ }^{20}$ and Somatic Indel Detector ${ }^{19}$ using matched normals. For the exomes we used VarScan ${ }^{21}$. All identified variants were annotated using Annovar ${ }^{22}$, and manually reviewed on IGV. Variants found in 1000 Genomes Project ${ }^{23,24}$, ESP6500, or dBSNP142 ${ }^{25}$ were excluded, unless also documented in COSMIC ${ }^{26}$. Significance for pathway enrichment was calculated via IPA (Ingenuity Systems) Ingenuity Pathway Analysis software and ToppGene, with both corrected by the Benjamini Hochberg procedure. Identified mutations were validated on MiSeq platform, followed by manual IGV review in eight PBs with sufficient material available for confirmation. Variant allele frequencies and coverage for validated variants are listed in the Supplementary Table 6. CNV analysis was performed using Control-FREEC ${ }^{27}$, an algorithm for detection of copy-number changes and allelic imbalances (including LOH) using deepsequencing data. Control-FREEC automatically computes, normalizes, segments copy number, and beta allele frequency (BAF) profiles, then calls copy-number alterations and $\mathrm{LOH}$.

Droplet digital PCR (ddPCR). Droplet digital PCR was performed on a Bio-Rad QX200 to verify the CNV region of chromosome 1q22. Primers were designed against regions of amplification for PDE4DIP and SEC22B and unamplified regions of HFE2 in the same chromosomal region. The RRP30 gene (diploid) was used as copy-number control, and normal pineal gland retrieved at autopsy as a normal tissue control. Primers will be provided upon request. A $20 \mathrm{ng}$ of HindIII digested genomic DNA were used per reaction, using the following protocol: 1 cycle at $95^{\circ} \mathrm{C}$ for $5 \mathrm{~min}, 40 \mathrm{cycles}$ at $95^{\circ} \mathrm{C}$ for $15 \mathrm{~s}$, and $60^{\circ} \mathrm{C}$ for $1 \mathrm{~min} ; 1$ cycle at $4{ }^{\circ} \mathrm{C}$ for $5 \mathrm{~min}$, and 1 cycle at $90^{\circ} \mathrm{C}$ for $5 \mathrm{~min}$ all at a ramp rate of $2{ }^{\circ} \mathrm{C} / \mathrm{s}$ on a Bio-Rad T100 thermal cycler was used for the PCR step. Droplets quantified using the Bio-Rad Quantisoft software. A total of two replicates were used per sample.

450k Array methylome clustering and copy-number profiling. DNA methylation was analyzed by the Illumina Infinium HumanMethylation450 (450k) array assessing 482,421 CpG sites (Illumina, San Diego, USA), according to the manufacturer's instructions at the NYU molecular laboratory. DNA methylation data were normalized by performing background correction and dye bias correction (shifting of negative control probe mean intensity to zero and scaling of normalization control probe mean intensity to 10,000 , respectively). Filtering of probes was performed as described previously ${ }^{4,28}$ (removal of probes targeting sex chromosomes, containing single nucleotide polymorphism and not uniquely matched). To enable future comparability, we also removed probes not represented on the novel Illumina Infinium HumanMethylation EPIC array. In total, 428,799 probes were kept for analysis. For unsupervised hierarchical clustering, we selected 5000 probes that showed the highest standard deviation across the beta values. Samples were hierarchically clustered using 1-Pearson correlation as a distance measure and average linkage as agglomeration method. The unscaled methylation levels were shown in a heat map from unmethylated state (blue color) to methylated state (red color). Copy-number profiles were generated using the "conumee" $\mathrm{R}$ package in Bioconductor (http://www.bioconductor.org/packages/release/bioc/html/conumee. $\mathrm{html})$, and assessed manually.

Whole-transcriptome sequencing and analysis. RNA was extracted from FFPE tissue using the automated Maxwell system (Promega, Madison, USA). RNASeq libraries were prepared using the Illumina TruSeq Stranded Total RNA library prep, with Ribozero Gold, starting from $500 \mathrm{ng}$ of DNAse I treated total RNA, following the manufacturer's protocol, with the exception that 14 cycles of PCR were performed to amplify the libraries, to keep the duplication rate lower than with the recommended 15 cycles. The amplified libraries were purified using AMPure beads, quantified by Qubit and QPCR, and visualized in an Agilent Bioanalyzer. The libraries were pooled equimolarly, and loaded at $8 \mathrm{pm}$, on a high output HiSeq 2500 flow cell, as paired 50 nucleotide reads. Sequencing results were demultiplexed and converted to FASTQ format using Illumina bcl2fastq software. The sequencing reads were aligned to the human genomes (build hg19/GRCh37) using the splice-aware STAR aligner ${ }^{29}$. The featureCounts program ${ }^{30}$ was utilized to generate counts for each gene based on how many aligned reads overlap its exons. These counts were then normalized and used to test for differential expression using negative binomial generalized linear models implemented by the DESeq2 R package ${ }^{31}$.

miRNA analysis. Extracted RNA quality and quantity were analyzed on an agilent Bioanalyzer 2100 using a nano chip. miRNA was hybridized using the Nanostring nCounter ${ }^{\circledast}$ Human v3A miRNA Expression Assay, according to the manufacturer's protocol. A total of $100 \mathrm{ng}$ RNA were used per sample. Hybridizations were processed on the nCounter Prep Station, and prepped cartridges were scanned on the Nanostring Digital Analyzer using 280 field of view counts. Data were analyzed with Nanostring nSolver 3.0. Samples were analyzed using the default quality control measures, and normalized to ligation controls in the Nanostring codesets.

Immunohistochemistry. Unconjugated polyclonal rabbit anti-human DUF1220 (Millipore Cat\# AB15369 Lot\# 2479814 RRID:AB_11214420) generated against a synthetic peptide from primates; and unconjugated polyclonal rabbit anti-human cardiomyopathy-associated protein 2 (PDE4DIP, Abcam Cat\# ab121375 Lot\# GR191210-1 RRID:AB 11128871) raised against a recombinant fragment, corresponding to amino acids 26-171 of human PDE4DIP isoform 2, were used for immunohistochemistry. Paraffin-embedded tissues were sectioned at $4-\mu \mathrm{m}$. Chromogenic immunohistochemistry was performed on a Ventana Medical Systems Discovery XT instrument with online deparaffinization using Ventana's reagents and detection kits unless otherwise noted. PDE4DIP was antigen retrieved in Ventana Cell Conditioner 1 (Tris-Borate-EDTA), and DUF1220 was antigen retrieved in Ventana Cell Conditioner 2 (Citrate) for $20 \mathrm{~min}$, respectively. Both antibodies were diluted 1:100 in Dulbecco's phosphate buffered saline (Invitrogen/ Life Technologies), and incubated for $3 \mathrm{~h}$ at room temperature. Primary antibody was detected with anti-rabbit, horseradish peroxidase conjugated multimer incubated for $8 \mathrm{~min}$. The complex was visualized with 3,3 diaminobenzidene and enhanced with copper sulfate. Slides were washed in distilled water, counterstained with hematoxylin, dehydrated, and mounted with permanent media. Negative controls were incubated with Dulbecco's phosphate buffered saline instead of primary antibody and did not show any signal. Immunohistochemistry was performed on 12 PB samples for which FFPE material was available and 15 normal control samples, 5 from fetal autopsy and 5 from adult fetopsy brains and their respective pineal glands. Tissue from fetal pineal glands were not available for analysis.

\section{Generation and validation of DROSHA knock-down in hNSC.}

Targeting DROSHA with LentiCRISPR: Genetic disruption of DROSHA was achieved by cloning small guide RNAs (sgRNAs), targeting DROSHA into lentiCRISPR-V2 vector (Addgene Plasmid \#52961). Briefly, oligonucleotides for sgRNAs were synthesized by Integrated DNA Technologies, annealed in vitro and ligated into a Bsmb1 digested lentiCRISPR-V2 vector (sgRNA: Sense-CACC G TACAGATCTGATTATGACCG, antisense-AAAC CGGTCATAATCAGATCTGTAC). The product was then used to generate lentivirus-containing supernatant that was concentrated by ultracentrifugation at $25000 \mathrm{xg}$ for $2 \mathrm{~h}$ at $4 \mathrm{C}$ ( $40 \mathrm{~mL}$ total). Concentrated virus was resuspended in $50 \mu \mathrm{L}$ of PBS.

Cell culture, transduction, and selection: hNSC were generated and maintained as in Pourchet et $\mathrm{al}^{32}$. These hNSCs have been characterized by RNA-seq, $450 \mathrm{~K}$ array DNA methylation profiling, and karyotype ${ }^{33}$. They are functionally multipotent, and can be directed to differentiate to neuroglial lineages. Briefly, hNSC were cultured in media supplemented with EGF and FGF2 and maintained on polyornithine/laminin coated plates. Cultures were routinely tested for mycoplasma 
contamination. One well in a six-well plate of hNSC were transduced with $2-8 \mu \mathrm{L}$ of packaged lentiCRISPR-V2 per well for $10 \mathrm{~h}$, at which time cells were washed and media changed every $48 \mathrm{~h}$. Seventy-two hours later, cells were cultured in $0.3 \mu \mathrm{g} /$ $\mathrm{mL}$ of puromycin for 7 days to generate only puromycin-resistant colonies. After selection, cells were passaged in $0.1 \mu \mathrm{g} / \mathrm{mL}$ of puromycin and then maintained in the absence of puromycin. This lentiCRISPR targeting of DROSHA was performed in triplicate.

Western blot: Successful Lenti-Cas9 expression and DROSHA knockdown were confirmed by Western blot. Protein lysate was prepared from Wild type (WT) and the triplicate DROSHA LentiCRISPR transduced hNSC lines using an $8 \mathrm{M}$ urea, $1 \%$ CHAPS buffer. Twenty micrograms of protein lysate was run per lane and resolved on a $4-12 \%$ Bis-Tris gel. After transfer, membranes were probed using antibodies directed at Cas9 (1:1000; Millipore MAC133), DROSHA (1:1000; Abcam 183732), GAPDH (1:5000; GeneTex 627408), H3 (1:5000; Abcam 1791). Visualization was performed using HRP-conjugated secondary antibodies directed against the appropriate primary antibody host, as developed using chemiluminescence. Average band intensity in each lane was measured for DROSHA, GAPDH, and histone $\mathrm{H} 3$ using ImageJ software. DROSHA intensity was normalized to the average of the GAPDH and $\mathrm{H} 3$ control band intensities.

Sanger sequencing: Primers were designed to amplify a $217 \mathrm{bp}$ fragment of the DROSHA locus that encompassed the gRNA target in Exon 4 (FWD Primer: CTATGATGACCACAGGCACCGA, REV Primer:

GGCCTCTGCAGTTCATTAAAG). Following standard qPCR amplification of this product, Sanger sequencing was performed for each of the three LentiCRISP hNSC lines generated.

Data availability. All original sequencing reads have been deposited in NCBI's public repository Sequencing Reads Archive (SRA). They are available under the BioProject ID: PRJNA446027.

In particular, the bam files are available under the accession ids SAMN08805007, SAMN08805008, SAMN08805009, SAMN08805010, SAMN08805011, SAMN08805012, SAMN08805013, SAMN08805014, SAMN08805015, SAMN08805016, SAMN08805017, SAMN08805018, SAMN08805019, SAMN08805020, SAMN08805021, SAMN08805022, SAMN08805023, SAMN08805024, SAMN08805025, and SAMN08805026.

No restrictions have been placed on data availability. Data should be available immediately after processing (by SRA).

Received: 22 December 2016 Accepted: 10 May 2018

Published online: 20 July 2018

\section{References}

1. de Kock, L. et al. Germ-line and somatic DICER1 mutations in pineoblastoma. Acta Neuropathol. 128, 583-595 (2014).

2. Han, S. J., Clark, A. J., Ivan, M. E., Parsa, A. T. \& Perry, A. Pathology of pineal parenchymal tumors. Neurosurg. Clin. N. Am. 22, 335-340, vii. (2011).

3. Louis D. N., Ohgaki H., Wiestler O. D. \& Cavenee W. K. WHO classification of tumours of the central nervous system, World Health Organization, International Agency for Research on Cancer, Revised 4th edition, (IARC Press, Lyon, France, 2016).

4. Sturm, D. et al. New brain tumor entities emerge from molecular classification of CNS-PNETs. Cell 164, 1060-1072 (2016).

5. Fevre-Montange, M. et al. Microarray analysis reveals differential gene expression patterns in tumors of the pineal region. J. Neuropathol. Exp. Neurol. 65, 675-684 (2006).

6. Fevre-Montange, M., Vasiljevic, A., Champier, J. \& Jouvet, A. Histopathology of tumors of the pineal region. Future Oncol. 6, 791-809 (2010).

7. Hovestadt, V. et al. Robust molecular subgrouping and copy-number profiling of medulloblastoma from small amounts of archival tumour material using high-density DNA methylation arrays. Acta Neuropathol. 125, 913-916 (2013).

8. Rakheja, D. et al. Somatic mutations in DROSHA and DICER1 impair microRNA biogenesis through distinct mechanisms in Wilms tumours. Nat. Commun. 2, 4802 (2014)

9. Wegert, J. et al. Mutations in the SIX1/2 pathway and the DROSHA/DGCR8 miRNA microprocessor complex underlie high-risk blastemal type Wilms tumors. Cancer Cell. 27, 298-311 (2015).

10. Torrezan, G. T. et al. Recurrent somatic mutation in DROSHA induces microRNA profile changes in Wilms tumour. Nat. Commun. 5, 4039 (2014).

11. O'Bleness, M. et al. Finished sequence and assembly of the DUF1220-rich 1q21 region using a haploid human genome. BMC Genom. 15, 387 (2014).

12. Uys, G. M. et al. Myomegalin is a novel A-kinase anchoring protein involved in the phosphorylation of cardiac myosin binding protein C. BMC Cell. Biol. 12, 18 (2011).
13. Brunetti-Pierri, N. et al. Recurrent reciprocal 1q21.1 deletions and duplications associated with microcephaly or macrocephaly and developmental and behavioral abnormalities. Nat. Genet. 40, 1466-1471 (2008).

14. Dumas, L. J. et al. DUF1220-domain copy number implicated in human brain-size pathology and evolution. Am. J. Hum. Genet. 91, 444-454 (2012).

15. O'Bleness, M. S. et al. Evolutionary history and genome organization of DUF1220 protein domains. G3 (Bethesda) 2, 977-986 (2012).

16. Popesco, M. C. et al. Human lineage-specific amplification, selection, and neuronal expression of DUF1220 domains. Science 313, 1304-1307 (2006)

17. Kim, Y. K., Kim, B. \& Kim, V. N. Re-evaluation of the roles of DROSHA, Export in 5, and DICER in microRNA biogenesis. Proc. Natl. Acad. Sci. USA 113, E1881-E1889 (2016)

18. Li, H. \& Durbin, R. Fast and accurate short read alignment with BurrowsWheeler transform. Bioinformatics 25, 1754-1760 (2009).

19. McKenna, A. et al. The Genome Analysis Toolkit: a MapReduce framework for analyzing next-generation DNA sequencing data. Genome Res. 20, 1297-1303 (2010)

20. Larson, D. E. et al. SomaticSniper: identification of somatic point mutations in whole genome sequencing data. Bioinformatics 28, 311-317 (2012).

21. Koboldt, D. C. et al. VarScan: variant detection in massively paralle sequencing of individual and pooled samples. Bioinformatics 25, 2283-2285 (2009).

22. Wang, K., Li, M. \& Hakonarson, H. ANNOVAR: functional annotation of genetic variants from high-throughput sequencing data. Nucleic Acids Res. 38, el64 (2010).

23. Zhang, W. et al. Comparing genetic variants detected in the 1000 genomes project with SNPs determined by the International HapMap Consortium. J. Genet. 94, 731-740 (2015).

24. Buchanan, C. C., Torstenson, E. S., Bush, W. S. \& Ritchie, M. D. A comparison of cataloged variation between International HapMap Consortium and 1000 Genomes Project data. J. Am. Med. Inform. Assoc. 19, 289-294 (2012).

25. Sherry, S. T. et al. dbSNP: the NCBI database of genetic variation. Nucleic Acids Res. 29, 308-311 (2001)

26. Forbes, S. A. et al. COSMIC: exploring the world's knowledge of somatic mutations in human cancer. Nucleic Acids Res. 43, D805-D811 (2015).

27. Boeva, V. et al. Control-FREEC: a tool for assessing copy number and allelic content using next-generation sequencing data. Bioinformatics 28, 423-425 (2012).

28. Sturm, D. et al. Hotspot mutations in H3F3A and IDH1 define distinct epigenetic and biological subgroups of glioblastoma. Cancer Cell. 22, 425-437 (2012).

29. Dobin, A. et al. STAR: ultrafast universal RNA-seq aligner. Bioinformatics 29, 15-21 (2013)

30. Liao, Y., Smyth, G. K. \& Shi, W. featureCounts: an efficient general purpose program for assigning sequence reads to genomic features. Bioinformatics $\mathbf{3 0}$, 923-930 (2014).

31. Love, M. I., Huber, W. \& Anders, S. Moderated estimation of fold change and dispersion for RNA-seq data with DESeq2. Genome Biol. 15, 550 (2014).

32. Pourchet, A., Modrek, A. S., Placantonakis, D. G., Mohr, I., \& Wilson, A. C. Modeling HSV-1 latency in human embryonic stem cell-derived neurons. Pathogens 6, E24 (2017)

33. Modrek, A. S. et al. Low-grade astrocytoma mutations in IDH1, P53, and ATRX cooperate to block differentiation of human neural stem cells via repression of SOX2. Cell Rep. 21, 1267-1280 (2017).

\section{Acknowledgements}

This study was in part supported by grants from the Friedberg Charitable Foundation, the Sohn Conference Foundation and the Making Headway Foundation to M.S. and M. A.K. and the Alice and Thomas J. Tisch Brain Tumor Research Fund to J.G.G. The NYU Experimental Pathology Immunohistochemistry Core Laboratory and the Genome Technology Center are supported in part by the Laura and Isaac Perlmutter Cancer Center Support Grant; NIH/NCI P30CA016087 and the National Institutes of Health S10 Instrumentation Grants; NIH/ORIP S10OD01058 and S10OD018338. This research was funded in part through the NIH/NCI Cancer Center Support Grant P30 CA008748 to Memorial Sloan Kettering Cancer Center. The authors would like to thank William L. Carroll, MD for his critical review of the manuscript and Stephen Conley for help with figures.

\section{Author contributions}

M.S. and M.A.K. conceived the study. M.S., E.P. S.W., A.F., D.Z., S.L.S., S.L.G., J.H.W, J.G.G., S.A., M.K.R., C.G.E., S.M.P, D.T.W.J. and M.A.K. provided tissue and clinical data. D.Z., M.K.R., C.G.E., A.F. and M.S. analyzed pathological data. O.A., I.D., K.K. and A.H. generated all WES and WGS data and performed ddPCR and RNA profiling. A.H., I.D., K.K. and M.S. analyzed the genomic data. J.M.S., K.R., D.G.P., S.E. LeB., T.Y.P. performed cell line experiments. J.S., D.C. V.H., L.C., S.M.P and D.T.W.J. performed DNA methylation profiling and analysis. L.C. performed immunohistochemical studies. 
All authors participated in writing the manuscript. All authors reviewed and agreed with the final version of the manuscript.

\section{Additional information}

Supplementary Information accompanies this paper at https://doi.org/10.1038/s41467018-05029-3.

Competing interests: The authors declare no competing interests.

Reprints and permission information is available online at http://npg.nature.com/ reprintsandpermissions/

Publisher's note: Springer Nature remains neutral with regard to jurisdictional claims in published maps and institutional affiliations. (c) (i) Open Access This article is licensed under a Creative Commons Attribution 4.0 International License, which permits use, sharing, adaptation, distribution and reproduction in any medium or format, as long as you give appropriate credit to the original author(s) and the source, provide a link to the Creative Commons license, and indicate if changes were made. The images or other third party material in this article are included in the article's Creative Commons license, unless indicated otherwise in a credit line to the material. If material is not included in the article's Creative Commons license and your intended use is not permitted by statutory regulation or exceeds the permitted use, you will need to obtain permission directly from the copyright holder. To view a copy of this license, visit http://creativecommons.org/ licenses/by/4.0/.

(C) The Author(s) 2018 